\title{
REDUCING BEAM COUPLING IMPEDANCES IN SNS RING EXTRACTION KICKERS*
}

\author{
S.S. Kurennoy, LANL, Los Alamos, NM 87545, USA, \\ D. Davino and Y.Y. Lee, BNL, Upton, NY 11973, USA
}

\begin{abstract}
The Spallation Neutron Source (SNS) Accumulator ring extraction system [1] includes 14 modules of windowframe ferrite pulsing kicker magnets with the rise time of about 200 ns. Their contribution to the beam coupling impedances is a serious concern. The kicker impedances, as well as its deflecting magnetic field versus time, are studied using detailed 3-D MAFIA modeling. Various design options, external circuit resistances, and a range of ferrite permeabilities are explored. A kicker module with wide conductor windings around the ferrite behind the kicker current sheet suggests a significant reduction of the kicker transverse and longitudinal coupling impedances. This design provides a good extraction field performance, as demonstrated by electromagnetic simulations. Results of measurements for a small model are also presented.
\end{abstract}

\section{INTRODUCTION}

The fast extraction kicker magnets of the SNS ring have to extract the proton beam accumulated during 1-ms storage cycle. The stored beam in the ring forms a single 650 ns long bunch that should be extracted during a single turn. The extraction gap is about 300 ns, which sets an upper limit of $200 \mathrm{~ns}$ on the kicker field rise time. The high intensity of the SNS accumulated beam requires minimizing the beam coupling impedances of the vacuum chamber elements, in spite of a rather short storage time for the circulating beam. To reduce the longitudinal impedance, one usually introduces a pair of flux breaks by inserting conductor sheets into the ferrite at the symmetry points [2]. We propose another flux break in the form of a conductive winding around the ferrite core behind the kicker current sheet. The winding is connected in a figureeight fashion so that it does not impede the rise of the kicker field but prevents any circulating flux.

\section{MAFIA MODELING}

We use 3-D time-domain simulations with the MAFIA code package [3]. The kicker model is illustrated in Figs. 1-2. The current plates are $40-\mathrm{cm}$ long, and provide the beam aperture $w \times h=128 \times 144 \mathrm{~mm}$. The surrounding ferrite bricks have length $30 \mathrm{~cm}$; their thickness is $24 \mathrm{~mm}$ on the sides, and $16 \mathrm{~mm}$ on the top and bottom. The copper breaks in the ferrite are shorted to the chamber walls. The conductor winding forms one-turn loops around the top and bottom horizontal ferrite bricks, and these loops are connected in topology of 8 as shown in Fig. 2.

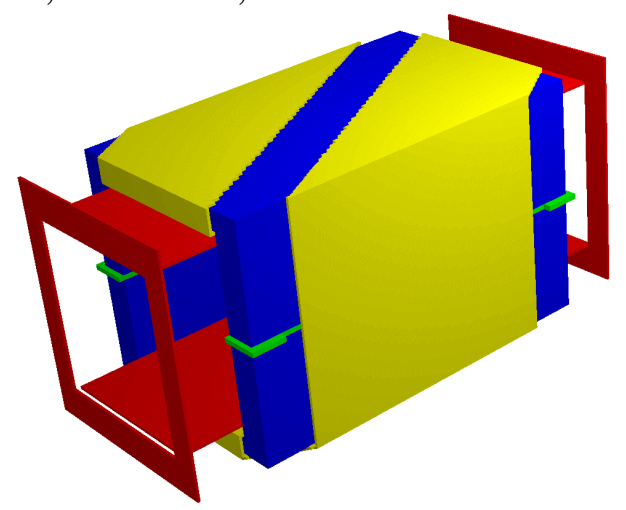

Figure 1: MAFIA model of SNS ring extraction kicker: ferrite (blue), current plates (red), breaks (green), and winding (yellow). Vacuum chamber is not shown.
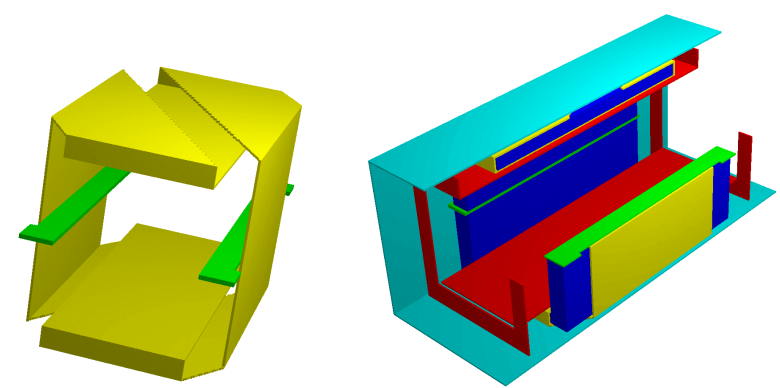

Figure 2: Model details: the winding and breaks (left), cutout view of the kicker inside the vacuum chamber.

The external PFN circuit is modeled by a resistor $R_{\text {ext }}$ in a current-plate gap (the left lower corner in Fig.1). For each set of model parameters like $R_{\text {ext }}$, ferrite permeability $\mu$ or its dielectric constant $\varepsilon$, we compute the longitudinal and transverse (with an off-axis beam) wake potentials of a long $(\sigma=10 \mathrm{~cm})$ Gaussian bunch using the time-domain module T3. Their Fourier transforms give us the beam coupling impedances. The wakes have to be long enough to provide a good resolution at low frequencies. Due to practical limitations, we compute wakes up to a certain length, typically $10 \mathrm{~m}$. It allows us to resolve frequencies above 15-30 MHz. The mesh inside the ferrite should be dense, because a typical value of $\varepsilon$ is 10 , and $\mu$ varies from 5 to 100 in simulations. For 1 -m long model structure, we end up with about 4.2M mesh points.

It is well known that in ferrites $\mu$ depends on frequency, quickly becoming quite large, on the order of 1000, at and below $1 \mathrm{MHz}$. This frequency dependence cannot be included in our MAFIA model; instead we just scan a range of $\mu$-values. However, above $10 \mathrm{MHz}$ where our results

*Work supported by Office of Basic Energy Science, the US Department of Energy, and by ORNL. 
for the impedances are applicable, the frequency dependence of $\mu$ is not so important. Some results for the beam coupling impedances are presented in Figs. 3 for a particular case of $R_{\text {ext }}=25 \Omega, \mu=40, \varepsilon=10$.

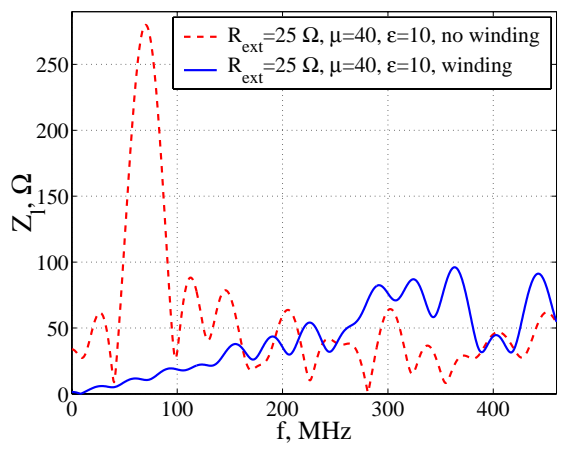

(a)
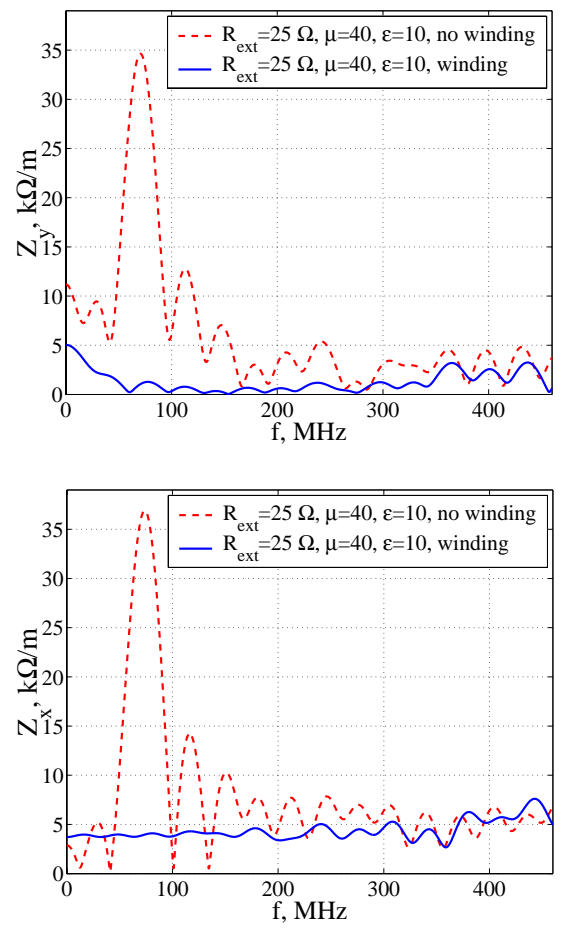

Figure 3: Kicker coupling impedances versus frequency, with (blue) and without the winding (red dashed): (a) longitudinal, (b) transverse vertical, (c) transverse horizontal.

It is obvious from Figs. 3 that the winding reduces the coupling impedances very effectively. The simulations show low-frequency resonances in the kicker model without the winding. Their frequencies slowly decrease as $\mu$ increases. A study of eigenmodes in the model with MAFIA eigensolver E leads to similar conclusions [4], as well as an analytical study of resonances in a long ferrite pipe [5]. However, the strong frequency dependence of $\mu$ at low frequencies can modify such predictions. In any case, the positive effect of the winding is clear. The longitudinal and transverse horizontal impedances behave as those of a typical inductance; their values are independent of $R_{\text {ext }}$ and slightly increase for larger $\mu$. The vertical transverse impedance at low frequencies shows some increase for larger $\mu$ and / or larger $R_{\text {ext }}$.
Another kind of time-domain simulations carried out with the model was computing the kicker deflecting magnetic field when a current was fed into the current plates. The current increases from 0 to its maximal value that was taken to be $3 \mathrm{kA}$, as time increases, with the rise time $200 \mathrm{~ns}$. The results for a few values of $\mu$ are presented in Fig. 4. Changing $R_{\text {ext }}$ influences only the field rise time.

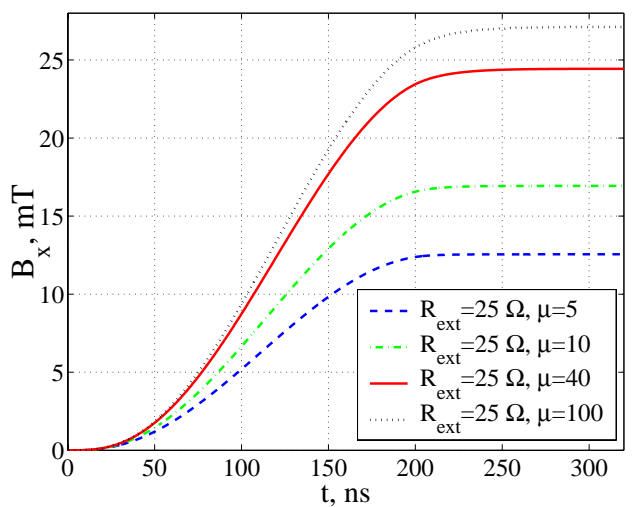

Figure 4: Kicker deflecting magnetic field versus time for various $\mu$ ( $R_{\mathrm{ext}}=25 \Omega, t_{\mathrm{rise}}=200 \mathrm{~ns}$, with the winding $)$.

The final field value is higher for larger $\mu$. Important to emphasize that curves for $B_{\mathrm{x}}(t)$ in the model without winding are practically identical to those in Fig. 4, so that the winding does not harm the kicker extraction performance. The extraction field quality is evident in Fig. 5, where the field snapshots in two planes are presented. The snapshots are taken at the moment 300 ns in Fig. 4 for $\mu=40$. The field uniformity is quite good, and becomes even better for larger permeabilities.

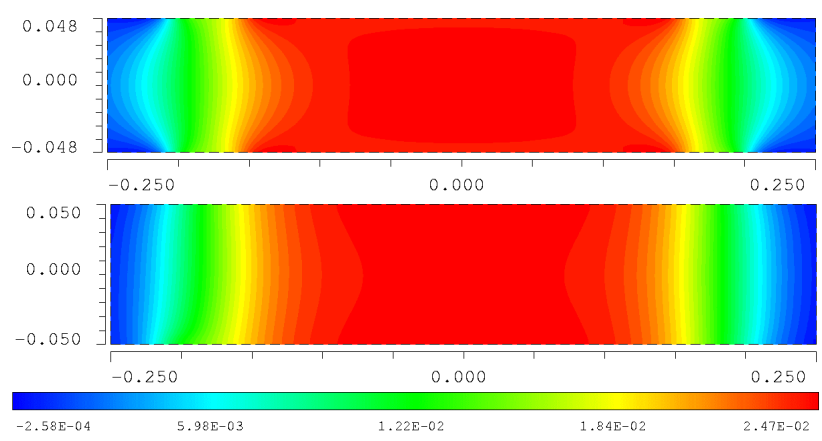

Figure 5: Contours of kicker deflecting magnetic field vs. longitudinal coordinate in the beam horizontal plane (top), and in the vertical one (middle). The scale is in T.

\section{MEASUREMENTS}

Transverse impedance measurements on a small model of the SNS extraction kicker have been carried out using the standard method [6], in which a twin-wire "Lecher" line is inserted into the kicker. The forward transmission coefficients $S_{21}$ of the "Device Under Test" and of a reference line of equal length are interpreted according to

$$
Z_{\perp}=\frac{c Z_{D U T}}{\omega \Delta^{2}}=-2 \frac{c Z_{L}}{\omega \Delta^{2}} \ln \left(S_{21 D U T} / S_{21 R E F}\right),
$$


with $\Delta$ being the spacing of the two wires and $Z_{L}$ the line characteristic impedance. The measurements were made with the commercial $450-\Omega$ cable CQ551, which has a spacing of $\Delta=13 / 16 \mathrm{in}=2.064 \mathrm{~cm}$. Matching the $450-\Omega$ line to $50 \Omega$ is achieved by means of a transformer (North Hills 0600BB) with a center-tapped secondary winding, which serves as $180^{\circ}$ hybrid. A network analyzer, Agilent 8753ES, set for the logarithmic frequency range from 30 $\mathrm{kHz}$ to $100 \mathrm{MHz}, 1601$ points and $100 \mathrm{~Hz}$ bandwidth, was used. The transformers are rated only for frequencies above $100 \mathrm{kHz}$, and therefore measurement results at lower frequencies must be used with care.

The dimensions of our model $-h \times w=65 \times 60 \mathrm{~mm}$ for the current plates $(L=200 \mathrm{~mm})$, and $75 \times 70 \mathrm{~mm}$ for the ferrite aperture $(L=150 \mathrm{~mm})$ - are smaller than those of the actual kicker. The ferrite thickness is $25 \mathrm{~mm}$. Our goal was to understand the behavior of the involved quantities, and to confirm the trends observed in MAFIA simulations. The size of the model implied noisy measurements in the $\mathrm{MHz}$ range, which is why the results here are presented only for frequencies above $5 \mathrm{MHz}$.

In Fig. 6, the transverse vertical impedances of the model without windings are compared for three terminations at the PFN port: $50 \Omega$, open, and short-circuit one. The open termination means that the PFN port is left as it is, which is roughly a very high resistance in parallel with a small capacitive impedance. One can see a factor of 2 difference between the impedances for the open and shortcircuit case. The short-circuit termination gives the lowest transverse impedance in the whole range of measured frequencies. The $50-\Omega$ case is always in the middle, except for the real part below $40 \mathrm{MHz}$ where the impedance is about $20 \%$ higher than for the open-circuit termination.

It is worth noting that the difference between open and short-circuit results depends strongly on the geometry and dimensions of measured kickers. Previous measurement on the AGS dump kicker [7] showed roughly a factor of 10 ; also see in reference [8].
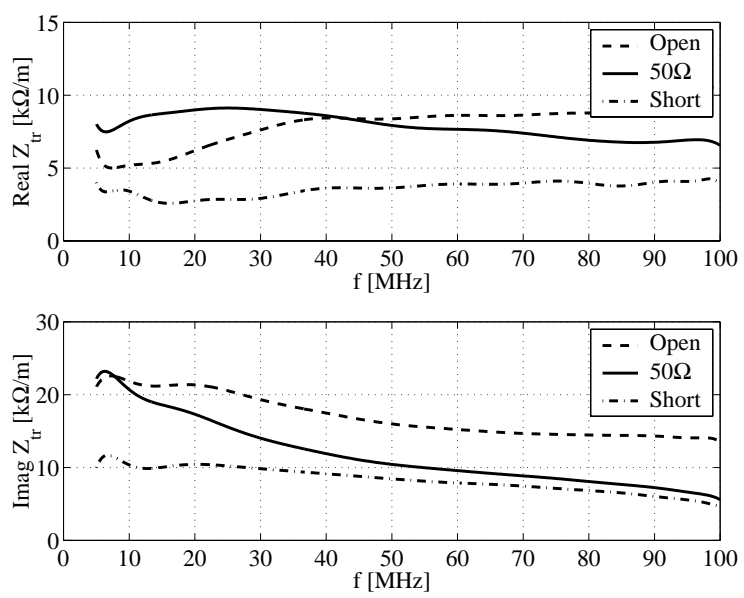

Figure 6. Transverse impedance of kicker model without ferrite winding: comparison of PFN-port terminations.

In Fig. 7, the transverse vertical impedances with the winding are shown. One can see a general reduction of the impedance magnitude by a factor of $2-1.25$ for all frequencies, compared to the case without winding. For the imaginary part, there is always a reduction of the transverse impedance, whereas for the real part of the opencircuit termination, below $20 \mathrm{MHz}$ the impedance is larger than without the ferrite winding.
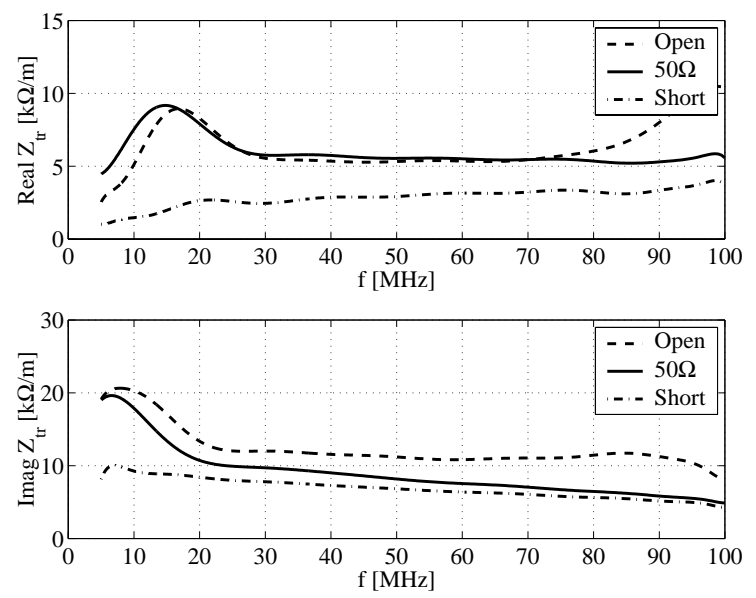

Figure 7. Transverse impedance of kicker model with ferrite winding: comparison of PFN-port terminations.

\section{CONCLUSIONS}

The MAFIA calculations for the SNS ring ferrite extraction kicker with the proposed winding show a significant reduction of both the transverse and longitudinal coupling impedances without affecting the kicker field or rise time. The measurements for a small kicker model also show the impedance reduction due to the winding, though less dramatic than in simulations.

Because of smaller dimensions of the measured model, a direct comparison between simulations and measurements is impossible, but the general trend of the impedance reduction with the winding is confirmed by the measurements. Measurements on the actual kicker that are planed in the near future will provide results for a quantitative comparison with the numerical modeling. We also plan to perform MAFIA simulations of the small model.

The authors would like to thank M. Blaskiewicz, V. Danilov, R. Gluckstern, and H. Hahn for useful discussions and advices.

\section{REFERENCES}

[1] N. Tsoupas, M. Blaskiewicz, Y.Y. Lee, et al. "Beam Extraction from the SNS Ring and Design of Extraction Kickers," EPAC00, Vienna, 2000, p. 2270.

[2] See, e.g., F. Voelker and G. Lambertson, Proceed. of PAC89, Chicago, 1989, p. 851.

[3] MAFIA, rel. 4.025. CTS (Darmstadt, Germany), 2000.

[4] V.Danilov, S.Kurennoy, M.Blaskiewicz, unpublished.

[5] R.L. Gluckstern, private communication.

[6] F. Caspers, in Handbook of Accelerator Physics and Engineering, ed. A.W. Chao and M. Tigner, p. 571.

[7] J.G. Wang and S.Y. Zhang, private communication.

[8] H. Hahn and D. Davino, these proceedings. 